\title{
Polidactilia en los cuatro miembros, en una potranca mestiza en Chile
}

\author{
Polydactyly in an all four limbs in a halfbred filly in Chile
}

\author{
Onésimo Sepúlveda S, MV, Christian Rehhof V, MV, Reinaldo Ortiz R, MV,
} Lisandro Muñoz A, M.Sc.*

Universidad de Concepción, Facultad de Ciencias Veterinarias, Departamento de Ciencias Clínicas, Av. Vicente Méndez 595, Chillán, Chile.*lismunoz@udec.cl

Recibido: Diciembre de 2010; Aceptado: Julio de 2011.

\section{RESUMEN}

La polidactilia es una anomalía congénita de muy baja incidencia en caballos. A una potranca mestiza de 6 semanas de edad con polidactilia en sus cuatro miembros se le realizó un examen clínico y radiológico. Los exámenes mostraron la presencia de un dígito supernumerario en ambos miembros anteriores, correspondiente a la primera y segunda falange del dígito II, con su respectivo hueso metacarpiano. En ambos miembros posteriores, se observó que un segundo dígito se originaba a nivel de la articulación metatarso-falángica; ambos dígitos eran del mismo tamaño y estaban formados por tres falanges, los que articulaban con el metatarsiano III. En ambos miembros posteriores, las primeras falanges estaban parcialmente fusionadas a nivel proximal. En el miembro posterior izquierdo había osteomielitis y fractura en el dígito lateral. Debido al compromiso del hueso y al dolor, se realizó eutanasia. No se realizó análisis citogenético. De acuerdo con la clasificación existente, la polidactilia encontrada en los miembros anteriores corresponde a la forma atavística y la encontrada en los posteriores a la forma teratogénica. Hasta lo que nosotros sabemos, este es el primer caso de polidactilia en los cuatro miembros registrado en América del Sur y en los últimos 55 años a nivel mundial.

Palabras clave: Anomalías congénitas, caballos, órganos supernumerarios. (Fuentes: AIMS, CAB). 


\section{ABSTRACT}

Polydactyly is a congenital anomaly of very low incidence in horses. A 6-week-old filly with polydactyly in all four limbs was admitted for a clinical and radiological examination. This examination showed the presence of a supernumerary digit for the digit II with respect to the metacarpal bone and the first and second phalanges in both forelimbs. In both hindlimbs a supernumerary digit was observed to originate at the level of the phalangic metatarsal joint. Both digits were the same size, and formed by three phalanges and were articulated with the metatarsi bone III. First phalanges of both digits were partially fused proximally. In the left hindlimb had osteomelitis, and a fracture in the lateral digit. Due to bone compromise and pain, euthanasia was performed. Cytogenic analysis was not performed. According to the used classification polydactyly described in the forelimbs corresponds to atavistic, and described in the hindlimbs to teratogenic form. As far as we know, this is the first case of polydactyly in all four limbs described in South America and the first in the world in the last 55 years.

Key words: Congenital abnormalities, horses, supernumerary organs. (Sources: AIMS, $C A B)$.

\section{INTRODUCCIÓN}

La polidactilia, se define como la presencia de uno o más dígitos extras (1). Aún cuando existen numerosos reportes, la polidactilia es poco frecuente, siendo estimada su incidencia en $0.066 \%$ (2). En Chile y América del Sur, no hay registro de polidactilia en equinos y a nivel mundial muy pocos de caballos con polidactilia en sus cuatro miembros, siendo el último en 1955, por lo que la descripción de este caso parece interesante considerando además, que es el primer caso en el que en un mismo individuo se presentan las 2 formas de polidactilia descritas.

\section{MATERIALES Y MÉTODOS}

Una potranca mestiza de 6 semanas de edad con polidactilia en sus cuatro miembros (Figura 1), ingresó junto a su madre al Hospital de Animales Mayores de la Facultad de Ciencias Veterinarias de la Universidad de Concepción, Sede Chillán, Chile, para una evaluación clínica y posible resolución quirúrgica del caso.

\section{RESULTADOS}

La anamnesis reveló que pese a las malformaciones, la potranca pudo ponerse de pie, caminar y alimentarse por si sola

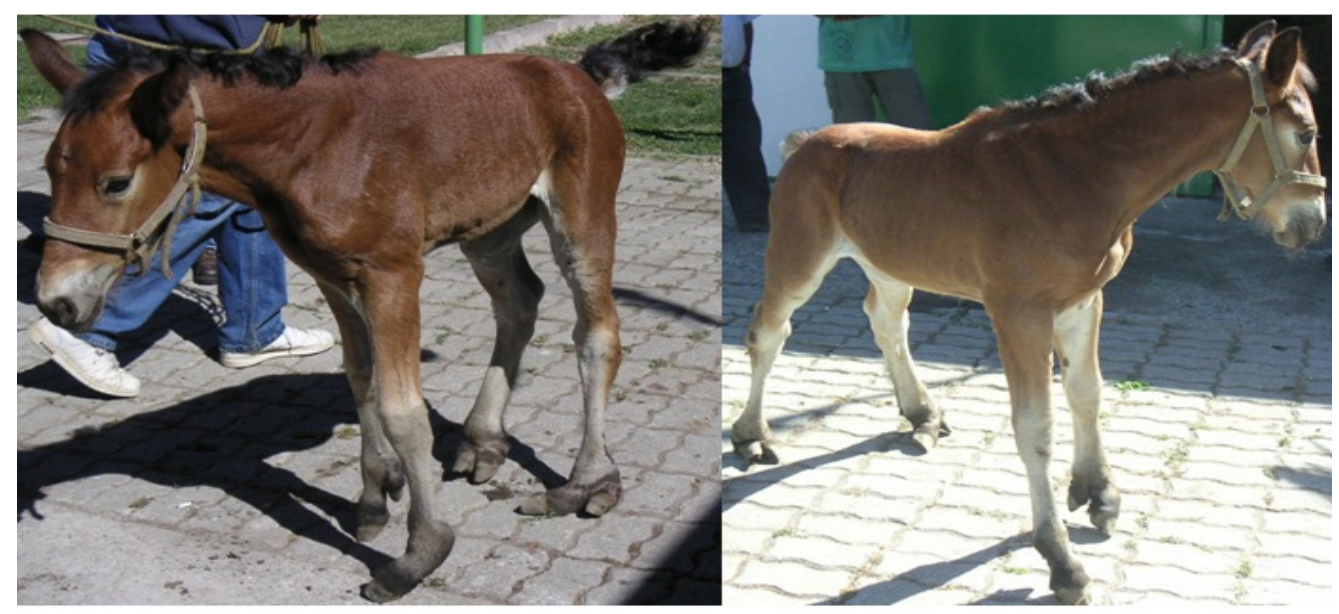

Figura 1. Vista lateral izquierda y derecha de la potranca con polidactilia en sus cuatro miembros. 
desde el primer día de vida, aunque a los pocos días comenzaron a hacerse más evidentes las deformidades angulares en el miembro anterior y posterior derecho, así como también, la deformidad flexural en los cuatro miembros, razón por la cual, en el último tiempo la potranca pasó la mayor parte del día en decúbito esternal o lateral.

Al examen clínico, durante la inspección a distancia, se observaba en ambos miembros anteriores aplomos de base ancha, deformidad flexural de la articulación interfalángica distal estado I, en que sólo el dedo del casco contactaba el suelo (apoyo en pinza) y polidactilia a partir del lado mediopalmar del nudo, en donde se originaba un dígito supernumerario completo con cuartilla y un casco de menor diámetro y tamaño que no tocaba el suelo y que alcanzaba aproximadamente hasta la mitad de la cuartilla del dígito III. Además, se observaba y palpaba el segundo hueso metacarpiano (Mc2), de menor diámetro que el Mc3. En el miembro anterior izquierdo el dígito III presentaba una desviación medial (valgus) severa a partir del nudo hasta tocar el suelo.

En los miembros posteriores, la polidactilia se manifestó en forma diferente, ya que por palpación se detectó el desarrollo del Mc2 rudimentario normal, observándose el nudo aumentado de volumen y del cual se originaban 2 dígitos con casco. En el miembro posterior derecho, la separación de los dígitos se producía a partir de la mitad de la cuartilla, siendo éstos de largo similar pero con el dígito medial de diámetro menor, en la vista frontal, los cascos se veían muy aguzados parecidos a las pezuñas de los rumiantes y en que el apoyo lo realizaba con la superficie plantar de la cuartilla y los talones del casco. Además, en la cerneja presentaba una herida abrasiva infectada de forma ovoide de $6 \times 4 \mathrm{~cm}$, de consistencia firme, con presencia de tejido de granulación y una depresión central en donde se encontró tejido necrótico y una fístula con abundante secreción de tipo serosanguinolenta.

En el miembro posterior izquierdo, el dígito medial era más largo que el lateral, y se encontraba totalmente luxado hacia la parte medial. El dígito lateral era más corto, con la cuartilla muy edematizada, pero debido a la luxación del dígito medial su casco tocaba el suelo y soportaba gran parte del peso del miembro. En la superficie lateral de este dígito había una herida abrasiva infectada de $6 \times 5 \mathrm{~cm}$, además, en la superficie dorsal sobre el rodete coronario había una fístula muy dolorosa a la palpación, que secretaba gran cantidad de secreción purulenta. En la superficie medial del dígito medial, había una herida de $4 \mathrm{~cm}$ de diámetro de consistencia firme. La potranca evidenciaba mucho dolor al caminar debido a la luxación y presencia de las heridas infectadas en sus miembros. La madre no presentaba polidactilia, además al ser consultado el propietario por antecedentes de polidactilia en el padrillo y crías del éste y/o la yegua, éste señaló que no tenía antecedentes.

Al examen radiológico, en los miembros anteriores se observó la presencia de un dígito supernumerario por medial (dígito II), con su respectivo hueso metacarpiano y la primera y segunda falange (P1, P2), el miembro anterior derecho (Figura 2) en el dígito supernumerario se observó sólo un hueso sesamoideo proximal, además la longitud de este dígito alcanzaba hasta

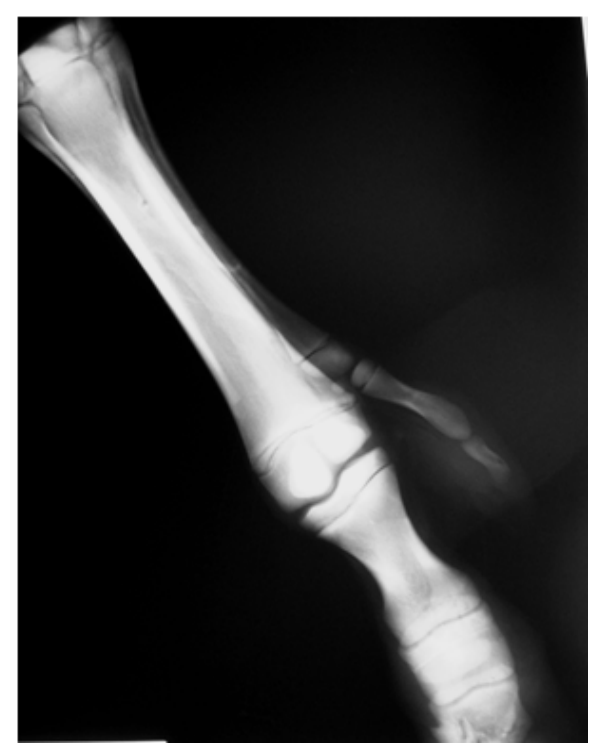

Figura 2. Radiografía dorsolateral-palmaromedial oblicua del metacarpo y dígitos del miembro anterior derecho. 
el nivel de la articulación interfalángica proximal del dígito III. En el miembro anterior izquierdo (Figura 3) el dígito supernumerario presentaba ambos huesos sesamoides proximales, y la longitud de este dígito alcanzaba hasta el nivel de la articulación interfalángica distal del dígito III.

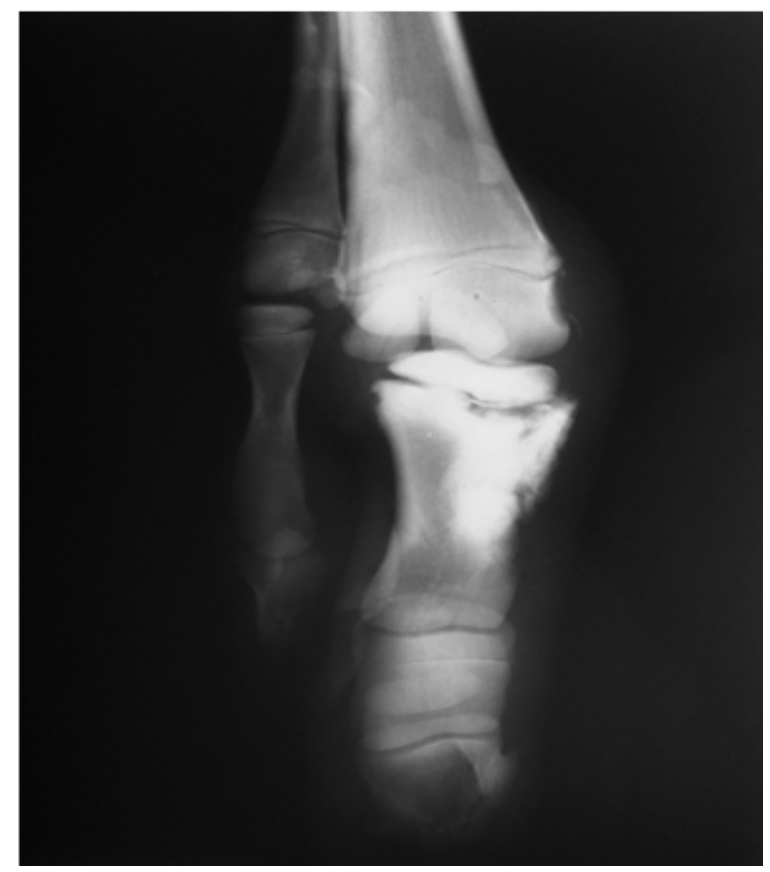

Figura 3. Radiografía dorsomedial-palmarolateral oblicua del nudo y falanges del miembro anterior izquierdo.

También, se observó una desviación del eje del dígito III hacia medial en la articulación metacarpofalángica, con desprendimiento lateral a nivel de la fisis del cartílago de crecimiento proximal de la P2 y una fractura de tipo Salter Harris 2, con signos de reparación, además, había signos de osteolisis en la P3 del dígito III. En ambos miembros posteriores, se observaba un dígito supernumerario, el cual se originaba a nivel de la articulación metatarsofalángica, ambos dígitos estaban formados por tres falanges y se articulaban con el hueso metatarsiano III (Mt3), uno en la carilla articular lateral y el otro en la medial, la P1 de ambos dígitos en los dos miembros se encontraban parcialmente fusionados en proximal. También se observaba que el crecimiento de ambos dígitos era del mismo tamaño hasta distal y que presentaban el hueso sesamoideo distal. No se pudo determinar cual era el dedo principal. En el miembro posterior derecho (Figura 4) la vista lateromedial permitió observar una deformación flexural

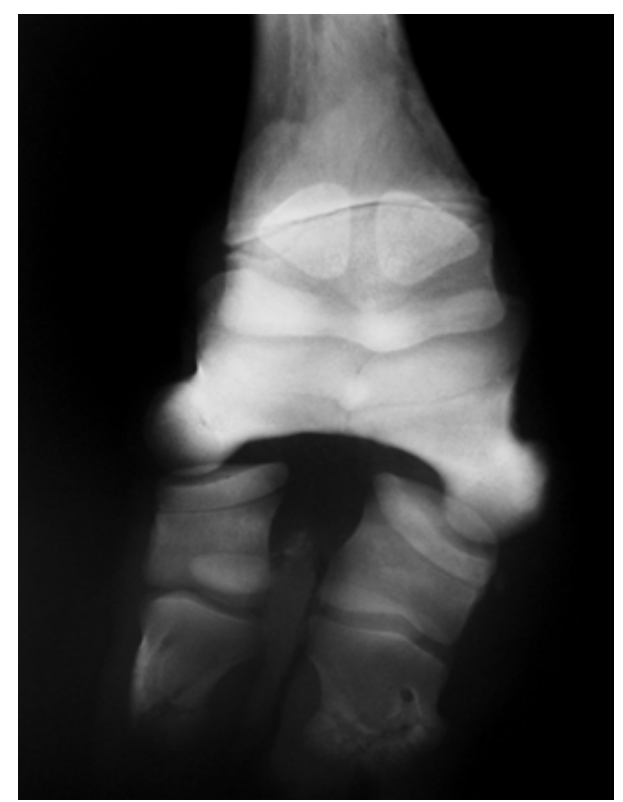

Figura 4. Radiografía dorsoplantar de las falanges del miembro posterior derecho.

marcada hacia plantar a nivel de articulación interfalángica proximal, por luxación completa de esta articulación en ambos dedos (hiperextensión). En el miembro posterior izquierdo (Figura 5) en la vista dorso plantar se observó pérdida del eje

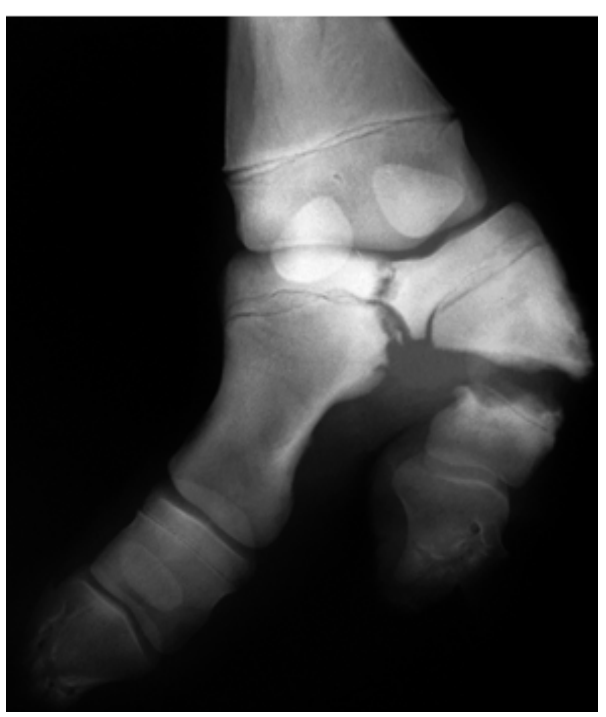

Figura 5. Radiografía dorsoplantar de las falanges del miembro posterior izquierdo. 
podofalángico normal visto de frente, con un quiebre hacia lateral (varus) en ambos dígitos y pérdida de arquitectura en ambas P3, especialmente en la zona del dedo. Además, se observó signos de osteolisis en la zona de fusión parcial de ambas P1. En el dígito lateral se observó una fractura de la primera falange, con signos de osteolisis severa. En la vista latero medial se observó una fractura de P1 en el dígito lateral, con signos de lisis ósea y presencia de aire o gas, concordante con osteomielitis.

Debido al compromiso óseo y dolor, se decidió realizar eutanasia. No se realizó análisis citogenético.

\section{DISCUSIÓN}

En 15224 necropsias de equinos realizadas en Estados Unidos, la adactilia y polidactilia mostraron tener una frecuencia muy baja de $0.066 \%$ (2). Ahora bien, la polidactilia en los cuatro miembros en equinos es menos frecuente, aún cuando en un estudio realizado por Lindermann en 1909, éste reportó que de 100 casos de polidactilia registrados en equinos, el $15 \%$ la presentaban en los 4 miembros (3). Sin embargo, desde el año 1909 hasta la fecha sólo se ha reportado un caso, el cual corresponde a una foto publicada en Alemania en 1955 (4). Diversas publicaciones revelan que son más frecuentes los casos de polidactilia medial y unilateral en los miembros anteriores (1,5$8)$, aún cuando, hay 3 casos de polidactilia bilateral en los miembros anteriores (3, $6,9)$ y otro de polidactilia bilateral en los posteriores (2).

Estos registros coinciden en cierta forma con las frecuencias de polidactilia reportadas por Lindermann en 1909, quien señaló que el $60 \%$ afectaban sólo a un miembro y el $24 \%$ a dos miembros (3). También, existen reportes de casos de polidactilia asociada a otras malformaciones congénitas como adactilia (8) y malformación craneal (2). La polidactilia ha sido reportada en diferentes razas y tipos de caballos entre ellas árabe, pura sangre, appalloosa, cuarto de milla, murgés, sangre templada alemán, poni Welsh, poni balcanés y también mestizo como el presente caso $(1,3,5,7-8,10)$. Se han reportado casos de polidactilia en Europa, Norte América y Australia (1-5, 7, $9,10)$, por lo que este caso de polidactilia, es el primero publicado en América del Sur.

La forma de polidactilia observada en este caso en los miembros anteriores, en que se hay desarrollo completo del Mc2 y su dígito, es similar a lo descrito por otros autores $(1,3,8,10)$, cuya corrección quirúrgica consiste en la extirpación del Mc2 y su dígito supernumerario, la que ha sido reportada con éxito por varios autores $(3,6,10)$.

La descripción de la polidactilia en los miembros posteriores, en que se observa la fusión parcial de las P1 con el desarrollo de 2 dígitos similares articulados al extremo distal del Mc3 es parecida a lo reportado en 2 equinos $(6,8)$, sin embargo, las fracturas, luxaciones $y$ osteolisis encontrada en estos dígitos no hicieron recomendable la corrección quirúrgica descrita por otros autores (6).

Lamentablemente, en este caso al no hacer un análisis citogenético no se pudo establecer o descartar si existía un problema a nivel de cromosomas. Sin embargo, de acuerdo a la clasificación propuesta por Stanek y Hantak (3) y posteriormente aceptada por Barber (5), la polidactilia descrita en los miembros anteriores corresponde a la forma atavística la que es definida como la formación de dígitos similares al dígito III presentes en antecesores y en que los dígitos supernumerarios usualmente se desarrollan desde la parte distal de los huesos metacarpianos o metatarsianos II y/o IV. Se entiende por atavismo la reaparición de caracteres de antepasados.

En la evolución del equino su primer antepasado el Hyracotherium (=Eohippus) hace 55 millones de años poseía 4 dígitos en los miembros anteriores y 3 en los miembros posteriores que tocaban el suelo, los que evolucionaron y fueron regresando paulatinamente hasta hace 10 millones de años, en que el Pliohippus fue el primer antepasado del equino actual que sólo poseía un dígito desarrollado con los metacarpales 
y metatarsales II y IV rudimentarios (11). Este caso correspondería a este tipo de polidactilia e incluso es similar fenotípicamente a los casos confirmados cromosomalmente por otros autores como forma atavísitica $(2,3)$. Respecto a lo observado en los miembros posteriores, los hallazgos corresponden a lo clasificado como polidactilia de forma teratógénica, ya que, los dígitos supernumerarios resultan de la división de los elementos basipodales, en que la mayoría de los casos tienen todos sus huesos falángicos afectados y en que frecuentemente los huesos metacarpales o metatarsales II y IV son normales (3, $5)$, caso muy similar al reportado por Dore (7) y clasificado por este autor como de origen teratogénico, entendiendo como tal la malformación producida por factores ambientales durante el desarrollo embrionario o fetal (11). Es poco probable pero no imposible, que en un mismo caso se produzca polidactilia de diferentes tipos. Lo más probable es que este caso sea de origen atavístico, atribuible como señalan algunos autores, a una mutación autosomal dominante con penetración incompleta $(1,3)$, por lo que esta polidactilia tendría un origen hereditario, lo cual también fue considerado por otros autores (10) al recomendar no reproducir el potro con polidactilia por ellos reportado, ya que se trataba de una malformación que se transmitía genéticamente.

En conclusión, la polidactilia en los cuatro miembros es de muy baja presentación y esta, es la primera reportada en Chile y América del Sur. Debido a la dificultad de clasificación de éste caso de polidactilia, parece necesario realizar futuros estudios de tipo cromosomal, para definir si la forma clasificada como teratogénica es tal.

\section{REFERENCIAS}

1. Giofré $F$, Caracciolo V, Zanotti M, Polli $M$, De Giovanni AM. Polydactyly in a murgese horse: a case report. J Equine Vet Sci 2004; 24(6):248-250.

2. Crowe, MW, Swerczek TW. Equine congenital defects. Am J Vet Res 1985; 46(2):353-358.

3. Stanek, C, Hantak E. Bilateral atavistic polydactyly in a colt and its dam. Equine Vet J 1986; 18(1):76-78.

4. Reinhardt OV. Pferd mit polydaktylie an allen vier füßen. Dtsch Tierärztl Wschr 1955; 62(11/12):109.

5. Barber SM. Unusual polydactylism in a foal. A Case Report. Vet Surg 1990; 19(3):203-207.

6. Carstanjen B, Abitbol M, Desbois C. Bilateral polydactyly in a foal. J Vet Sci 2007; 8(2):201-203.

7. Dore MAP. Teratogenic polydactylism in a halfbred foal. Vet Rec 1989 125:375-376.
8. Leipold HW, MacDonald KR. Adactylia and polydactylia in a Welsh foal. Vet Med Small Anim Clin 1971; 66:298-230.

9. Novales MD, Hernández RP, Sánchez de Medina A, Lara F, Muñoz EP. Signos radiológicos de la polidactilia en potros. En: XV Congreso Chileno de Medicina Veterinaria. Pucón: 2008.

10. Weinhart G, Götz E, Götz H-J. Polydactylie beim fohlen-ein fallbericht. Tierärztl Prax 1996; 24: 275-277.

11. Bowling AT, Ruvinsky A. Genetics aspects of domestication, breeds and their origins. En: Bowling AT, Ruvinsky A. dir. The genetics of the horse. London: CAB International; 2000. 